\title{
Canonical Forms and Their Integrability for Systems of Three 2nd-Order ODEs
}

\author{
S. Zahida, ${ }^{1}$ M. N. Qureshi, ${ }^{1}$ and Muhammad Ayub ${ }^{2}$ \\ ${ }^{1}$ Department of Mathematics, Azad Jammu and Kashmir University, Muzaffarabad, Pakistan \\ ${ }^{2}$ Department of Mathematics, COMSATS Institute of Information Technology, Abbottabad, Pakistan \\ Correspondence should be addressed to Muhammad Ayub; muhammad_ayub5@hotmail.com
}

Received 5 April 2017; Accepted 25 May 2017; Published 16 July 2017

Academic Editor: Mariano Torrisi

Copyright (C) 2017 S. Zahida et al. This is an open access article distributed under the Creative Commons Attribution License, which permits unrestricted use, distribution, and reproduction in any medium, provided the original work is properly cited.

Differential invariants and their corresponding canonical forms for systems of three 2nd-order ODEs possessing three-dimensional Lie algebras are constructed. Their extension up to $k$ th-order system of three 2 nd-order ODEs is presented. Furthermore singularity in invariant structure for the canonical forms is investigated. In addition integrability of these canonical forms is discussed. Illustrative physical examples from mechanics of system of particles are provided.

\section{Introduction}

There are several physical phenomena whose mathematical modeling is associated with system of 2nd-order ODEs and hence analysis of various aspects of these systems of ordinary differential equations plays a vital role in the applied sciences. Due to this contributing role and importance, these systems have been extensively studied over the years. Different methods and approaches have been introduced and constructed for various aspects of the analysis of these systems.

One of the prominent approaches is Lie symmetry method, initiated by Sophus Lie [1842-1899]. This is a general method in which exact solutions and various other aspects of linear and nonlinear differential equations are analysed. Lie theory is based on Lie groups and the corresponding Lie algebras. Lie proved that the integrability of a differential equation is contingent on the properties of the Lie algebra it admits; for details see [1-5].

In Lie theory, there are two approaches for the integrability of differential equations: direct and indirect approach. In the direct approach classical reduction is performed via differential invariants and canonical variables and so forth, while in the indirect approach equations are classified via their admitted Lie algebras in prescribed number of underlying variables with corresponding set of structure constants.
Then algebraic realizations are used for integration of these classified forms. The indirect approach is also known as Lie algebraic approach. The direct approach is useful for scalar ordinary differential equations only but does not work for system of ordinary differential equations [6]. The indirect approach is equally valid for both scalar ODEs as well as system of ordinary differential equations.

In this paper, we are interested in Lie algebraic approach (Realization approach) for system of 2nd-order ordinary differential equations.

To apply the algebraic approach, equations have to be classified according to their admitted symmetry algebras. Moreover this classification scheme depends not only upon the abstract Lie algebras (and their subalgebras) but also the realizations of the admitted Lie algebras in prescribed number of variables.

The initial work on classification and realizations of Lie algebras was done by Lie [7]. After Lie, several researchers have been involved in analysis of different aspects of this work; see $[1,6,8-12]$. Although the algebraic approach is more involved, it generates new cases and more insight to mathematical properties whose base is algebra (see $[8$, $9,13,14])$. Thus the classification of differential equations via algebraic approach is more efficient than other classical approaches. Unluckily, very limited literature is available on 
this approach for system of 2nd-order ordinary differential equations.

Like systems of two 2nd-order ODEs, systems of three 2nd-order ODEs have vital role in different mathematical modeling of physical situations such as small oscillation problems, wave propagation problems, and problems of mechanics. There is very limited and restricted work on classification of system of three 2nd-order ODEs according to their symmetry algebras (see $[15,16])$. Thus in this paper, we are interested in investigating classification of system of three 2nd-order ODEs via the algebraic approach for dimension 3.

The outline of the rest of the paper is as follows. In Section 2 invariant structure of canonical forms and their Lie algebraic properties are constructed. Section 3 is about the integrability of underlying canonical forms, whereas Section 4 presents illustrative examples of the results obtained in this paper. Paper ends with a brief conclusion.

\section{Differential Invariants and Canonical Forms}

By fixing number of prescribed variables for a given set of structure constants associated with an underlying Lie algebra one can obtain the most general classes of system of differential equations which admit the investigated Lie algebra in prescribed number of variables.

Here group classification of system of three 2 nd order ODEs, admitting three-dimensional Lie algebra, has been investigated via algebraic approach. The classification results of [11] are utilized but in the format of [8].

2.1. Invariant Construction. Following result given in [8] is fundamental in construction of invariants and their corresponding canonical forms.

Proposition 1. If $u$ and $v$ are invariants of a Lie algebra admitted by any system of ordinary differential equations, then $d v / d u$ is also its invariant.

For the construction of canonical forms the approach developed in $[8,9,13]$ is employed. By utilizing realizations of underlying 3-dimensional Lie algebras admitted by system of three 2nd-order ordinary differential equations we find differential invariants associated with them. These invariants are then used to construct invariant system of differential equations, called canonical forms of system.

Notations. The following notions are used in rest of the paper:

$\mathscr{A}_{i, j}^{a, b, n}$ denotes the $j$ th Lie algebra of dimension $i$ whereas superscripts indicate parameters on which the algebra possibly depends; the column $N$ in Table 1 gives the algebra realizations; the realization is referred to by a superscript $n$; as usual $\partial_{t}=\partial / \partial t, \partial_{x}=$ $\partial / \partial x, \partial_{y}=\partial / \partial y, \partial_{z}=\partial / \partial z$.

$X_{i}$ denotes the elements of a basis of a given Lie algebra; here $i$ is less than or equal to the dimension of the underlying real Lie algebra. The rank of the associated realization is denoted by $r$ and $N$ is the realization of the corresponding algebra.
The realizations given in [8] are used to obtain the differential invariants and their corresponding invariant representation for the system of three 2nd-order ordinary differential equations. There are various methods and approaches for finding differential invariants but we follow the peculiar approach developed by Ayub et al. in $[8,9,13]$. In this approach invariants are interrelated via basic invariant. We explain the algorithm by the following example.

Example 2. Consider the second realization $\mathscr{A}_{3,5}^{2} \simeq\left\langle\partial_{t}, \partial_{x}\right.$, $\left.t \partial_{t}+x \partial_{x}+\partial_{y}\right\rangle$ from [8]. We find the second prolongation of generators and then adopting the procedure of construction of differential invariants described in $[8,9,13]$, we obtain the following differential invariants:

$$
\begin{aligned}
p & =z, \\
q & =\dot{x}, \\
u & =\dot{y} e^{y}, \\
v & =\frac{\dot{z}}{\dot{y}}, \\
\frac{d q}{d p} & =\frac{\ddot{x}}{\dot{z}}, \\
\frac{d u}{d p} & =\dot{y} e^{y}\left(\frac{\dot{y}}{\dot{z}}+\frac{\ddot{y}}{\dot{y} \dot{z}}\right), \\
\frac{d v}{d p} & =\frac{\ddot{z}}{\dot{y} \dot{z}}-\frac{\ddot{y}}{\dot{y}^{2}} .
\end{aligned}
$$

This gives the following invariant representation and associated canonical form for the system of three 2nd-order ODEs admitting Lie algebra $\mathscr{A}_{3,5}^{2}$ :

$$
\begin{aligned}
\frac{d q}{d p} & =f(p, q, u, v) \\
\frac{d u}{d p} & =g(p, q, u, v) \\
\frac{d v}{d p} & =h(p, q, u, v) \\
\frac{\ddot{x}}{\dot{z}} & =f(p, q, u, v) \\
\dot{y} e^{y}\left(\frac{\dot{y}}{\dot{z}}+\frac{\ddot{y}}{\dot{y} \dot{z}}\right) & =g(p, q, u, v) \\
\frac{\ddot{z}}{\dot{y} \dot{z}}-\frac{\ddot{y}}{\dot{y}^{2}} & =h(p, q, u, v) .
\end{aligned}
$$

Here and in the subsequent work $p, q, u, v, d q / d p, d u / d p$, and $d v / d p$ denote invariants whereas $f, g$, and $h$ are arbitrary functions. 
Example 3. Consider the algebra $\mathscr{A}_{3,8}^{4} \simeq\left\langle-t \partial_{x},(1 / 2)\left(-t \partial_{t}+\right.\right.$ $\left.\left.x \partial_{x}\right), x \partial_{t}\right\rangle$ given in [8]. Proceeding as in above example we arrive at the following invariants:

$$
\begin{aligned}
p & =z, \\
q & =y, \\
u & =\frac{t \dot{x}-x}{\dot{y}}, \\
\frac{d q}{d p} & =\frac{\dot{y}}{\dot{z}}, \\
w & =\frac{\ddot{x}}{\dot{y}^{3}}, \\
\frac{d u}{d p} & =\frac{t \dot{x}}{\dot{y} \dot{z}}-\frac{\ddot{y}(t \dot{x}-x)}{\dot{y}^{2} \dot{z}}, \\
\frac{d^{2} q}{d p^{2}} & =\frac{\ddot{y}}{\dot{z}^{2}}-\frac{\dot{y} \ddot{z}}{\dot{z}^{3}} .
\end{aligned}
$$

Using these invariants we obtain the following invariant representation and corresponding canonical form for the system of three 2 nd-order ODEs possessing Lie algebra $\mathscr{A}_{3,8}^{4}$ :

$$
\begin{aligned}
& w=f\left(p, q, u, \frac{d q}{d p}\right) \\
& \frac{d u}{d p}=g\left(p, q, u, \frac{d q}{d p}\right) \\
& \frac{d^{2} q}{d p^{2}}=h\left(p, q, u, \frac{d q}{d p}\right) \\
& \frac{\ddot{x}}{\dot{y}^{3}}=f\left(p, q, u, \frac{d q}{d p}\right) \\
& \frac{t \dot{x}}{\dot{y} \dot{z}}-\frac{\ddot{y}(t \dot{x}-x)}{\dot{y}^{2} \dot{z}}=g\left(p, q, u, \frac{d q}{d p}\right) \\
& \frac{\ddot{y}}{\dot{z}^{2}}-\frac{\dot{y} \ddot{z}}{\dot{z}^{3}}=h\left(p, q, u, \frac{d q}{d p}\right) \text {. }
\end{aligned}
$$

In classification problem related to system of differential equations, invariant interrelation plays a key role in different types of analysis, namely, integrability, Linearization, and singularity as well as some other aspects especially related to system of three 2 nd-order ODEs (see $[8,9,13])$. By careful observations here we have obtained some relations between invariants. On the basis of these relations we categorize the regular differential invariants in the following main two types.

(i) Invariant Representation in First-Order System of ODEs. In this system, for underlying Lie algebra with peculiar realization, we obtain seven linearly independent invariants whose invariant representation is in the form of system of first-order ordinary differential equations. These invariants contain four first-order differential invariants and three second-order differential invariants. The four first-order differential invariants include one invariant that plays a role of basic invariant $(p)$. The second-order differential invariants are derived via differentiation property of first-order differential invariants with respect to the basic invariant, for example, $\mathscr{A}_{3,5}^{2}$.

(ii) Invariant Representation in Mixed Type System of 2ndOrder ODE. In this system, for underlying Lie algebra with peculiar realization, we obtain seven linearly independent invariants whose invariant representation is in the form of mixed type system of 2 nd-order ordinary differential equations. These invariants contain four first-order differential invariants and three 2nd-order differential invariants. The four first-order differential invariants include one invariant that plays a role of basic invariant $(p)$ and remaining three; one of the invariant is derived by the differentiation of another invariant from the remaining two with respect to the basic invariant. The 2nd-order differential invariants contain one independent invariant $(w)$ and the remaining two are obtained via differentiation of first-order differential invariants with respect to the basic invariant, for example, $\mathscr{A}_{3,8}^{4}$.

Both types of invariants are presented in Table 1. These observations led us to the following.

Remark 4. The number of functionally independent invariants for the regular system of three 2nd-order ODEs admitting three-dimensional Lie algebra is 7 .

2.2. Singularity in Invariant Structure. If the rank of solution manifold is less than the rank of generic manifold, then invariant differential equation is singular [13]. This singularity plays an important role in the integrability of such systems.

For example, consider the case of Lie algebra $\mathscr{A}_{3,1}^{3} \simeq$ $\left\langle\partial_{x}, t \partial_{x}, y \partial_{x}\right\rangle$. By employing the same procedure as used for the invariant construction of $\mathscr{A}_{3,5}^{2}$ and $\mathscr{A}_{3,8}^{4}$ in the previous subsection, the following set of differential invariants is found:

$$
\begin{aligned}
p & =t, \\
q & =y, \\
u & =z, \\
\frac{d q}{d p} & =\dot{y}, \\
\frac{d u}{d p} & =\dot{z}, \\
w & =\ddot{x}, \\
\frac{d^{2} u}{d p^{2}} & =\ddot{z}, \\
\frac{d^{2} q}{d p^{2}} & =0
\end{aligned}
$$


The corresponding invariant representation and canonical form for the system of three 2 nd-order ordinary differential equations having the Lie algebra $\mathscr{A}_{3,1}^{3}$, respectively, are

$$
\begin{aligned}
w & =f\left(p, q, u, \frac{d q}{d p}\right) \\
\frac{d^{2} q}{d p^{2}} & =0 \\
\frac{d^{2} u}{d p^{2}} & =h\left(p, q, u, \frac{d q}{d p}\right) \\
\ddot{x} & =f\left(p, q, u, \frac{d q}{d p}\right) \\
\ddot{y} & =0 \\
\ddot{z} & =h\left(p, q, u, \frac{d q}{d p}\right) .
\end{aligned}
$$

By employing the same procedure as given in [13], it is deduced that the condition of singularity is satisfied for the set of invariants given in (5) and hence possesses the singularity structure.

This type of singularity in invariant structure occurs in $\mathscr{A}_{3,5}^{3}$ presented in Table 1 .

If we consider the Lie algebra $\mathscr{A}_{3,1}^{4}$, we obtain the following set of differential invariants:

$$
\begin{aligned}
p & =t, \\
q & =y, \\
u & =z, \\
\frac{d q}{d p} & =\dot{y}, \\
\frac{d u}{d p} & =\dot{z}, \\
w & =\ddot{x}, \\
\frac{d^{2} u}{d p^{2}} & =\ddot{z}, \\
\frac{d^{2} q}{d p^{2}} & =0 \quad(\ddot{y}=0),
\end{aligned}
$$

whereas $\mathscr{A}_{3,1}^{4}$ and $\mathscr{A}_{3,8}^{1}$ do not form the system of three 2 ndorder ODEs.

2.3. Extension in System of Three kth-Order ODEs. Utilizing the interrelationship between invariants of three secondorder ODEs, three $k$ th-order ordinary differential equations admitting three-dimensional algebras can be constructed, as done in [8].

For this purpose we utilize the basic invariant that is introduced in the invariant representation for the classification. Consider the case of $\mathscr{A}_{3,8}^{4}$ that belongs to the invariant representation in mixed type system of 2 nd-order ODEs. We have the following invariant structure for this case:

$$
\begin{aligned}
p & =z, \\
q & =y, \\
u & =\frac{t \dot{x}-x}{\dot{y}}, \\
\frac{d q}{d p} & =\frac{\dot{y}}{\dot{z}}, \\
w & =\frac{\ddot{x}}{\dot{y}^{3}}, \\
\frac{d u}{d p} & =\frac{t \dot{x}}{\dot{y} \dot{z}}-\frac{\ddot{y}(t \dot{x}-x)}{\dot{y}^{2} \dot{z}}, \\
\frac{d^{2} q}{d p^{2}} & =\frac{\ddot{y}}{\dot{z}^{2}}-\frac{\dot{y} \ddot{z}}{\dot{z}^{3}} .
\end{aligned}
$$

Differentiating the set of second-order differential invariants with respect to basic invariant $(p)$, we find the following 3 rdorder differential invariants:

$$
\begin{aligned}
\frac{d w}{d p}= & \frac{\dddot{x}}{\dot{z} \dot{y}^{3}}-3 \frac{\ddot{x} \ddot{y}}{\dot{z} \dot{y}^{4}}, \\
\frac{d^{2} u}{d p^{2}}= & \frac{(t \ddot{x}+\dot{x})}{\dot{y} \dot{z}^{2}}-\frac{t \dot{x} \ddot{z}}{\dot{y} \dot{z}^{3}}-\frac{\ddot{y}(t \ddot{x}+\dot{x}(t-1))}{\dot{y}^{2} \dot{z}^{2}} \\
& +\frac{2 \ddot{y}^{2}(t \dot{x}-x)}{\dot{y}^{3} \dot{z}^{2}}+\frac{\ddot{y} \ddot{z}(t \dot{x}-x)}{\dot{y}^{2} \dot{z}^{3}}-\frac{\dddot{y}(t \dot{x}-x)}{\dot{y}^{2} \dot{z}^{2}}, \\
\frac{d^{3} q}{d p^{3}}= & \frac{\dddot{y}}{\dot{z}^{3}}-3 \frac{\ddot{y} \ddot{z}}{\dot{z}^{4}}+3 \frac{\dot{y} \ddot{z}^{2}}{\dot{z}^{5}}-\frac{\dot{y} \ddot{z}}{\dot{z}^{3}} .
\end{aligned}
$$

Thus the set of functionally independent invariants for the system of three 3rd-order ordinary differential equations possessing $\mathscr{A}_{3,8}^{4}$ is

$$
\begin{gathered}
p=z, \\
q=y, \\
u=\frac{t \dot{x}-x}{\dot{y}}, \\
\frac{d q}{d p}=\frac{\dot{y}}{\dot{z}}, \\
\frac{d u}{d p}=\frac{\ddot{x}}{\dot{y}}, \\
\frac{\dot{y}^{3}}{\dot{z}}-\frac{\ddot{y}(t \dot{x}-x)}{\dot{y}^{2} \dot{z}}, \\
\frac{d^{2} q}{d p^{2}}=\frac{\ddot{y}}{\dot{z}^{2}}-\frac{\dot{y} \ddot{z}}{\dot{z}^{3}},
\end{gathered}
$$


TABLE 1: 2nd-order differential invariants and equations.

\begin{tabular}{|c|c|c|}
\hline Algebra & $N$ & Invariants and equations \\
\hline$A_{3,1}$ & 2 & $\begin{array}{l}p=z, q=\dot{x}, u=\dot{y}, v=\dot{z}, \frac{d q}{d p}=\frac{\ddot{x}}{\dot{z}}, \frac{d u}{d p}=\frac{\ddot{y}}{\dot{z}}, \frac{d v}{d p}=\frac{\ddot{z}}{\dot{z}} \\
\ddot{x}=f(p, q, u, v), \frac{\ddot{y}}{\dot{z}}=g(p, q, u, v), \frac{\ddot{z}}{\dot{z}}=h(p, q, u, v) \\
p=t, q=z, u=\dot{y}-\dot{x} f^{\prime}(t), \frac{d q}{d p}=\dot{z}, w=\ddot{x}, \frac{d u}{d p}=\ddot{y}-\ddot{x} f^{\prime}(t)-\dot{x} f^{\prime \prime}(t), \frac{d^{2} q}{d p^{2}}=\ddot{z} \\
\ddot{x}=f\left(p, q, u, \frac{d q}{d p}\right), \ddot{y}-\ddot{x} f^{\prime}(t)-\dot{x} f^{\prime \prime}(t)=g\left(p, q, u, \frac{d q}{d p}\right), \ddot{z}=h\left(p, q, u, \frac{d q}{d p}\right) \\
p=t, q=y, u=z, \frac{d q}{d p}=\dot{y}, w=\ddot{x}, \frac{d u}{d p}=\dot{z}, \frac{d^{2} u}{d p^{2}}=\ddot{z} \& \frac{d^{2} q}{d p^{2}}=\ddot{y}=0 \\
\ddot{x}=f\left(p, q, u, \frac{d q}{d p}, \frac{d u}{d p}\right), \ddot{y}=0, \ddot{z}=h\left(p, q, u, \frac{d q}{d p}, \frac{d u}{d p}\right) \\
p=z, q=y, u=\frac{\dot{x}}{\dot{y}}-\frac{\dot{z}}{\dot{y}^{2}}, \frac{d q}{d p}=\frac{\dot{y}}{\dot{z}}, w=\frac{\ddot{y}}{\dot{y}^{3}}, \frac{d u}{d p}=\frac{\ddot{x}}{\dot{y} \dot{z}}-\frac{\ddot{z}}{\dot{y}^{2} \dot{z}}+\frac{\ddot{y}(2 \dot{z}-\dot{x} \dot{y})}{\dot{y}^{3} \dot{z}}, \frac{d^{2} q}{d p^{2}}=\frac{\ddot{y}}{\dot{z}^{2}}-\frac{\dot{y} \ddot{z}}{\dot{z}^{3}} \\
\frac{\ddot{y}}{\dot{y}^{3}}=f\left(p, q, u, \frac{d q}{d p}\right), \frac{\ddot{x}}{\dot{y} \dot{z}}-\frac{\ddot{z}}{\dot{y}^{2} \dot{z}}+\frac{\ddot{y}(2 \dot{z}-\dot{x} \dot{y})}{\dot{y}^{3} \dot{z}}=g\left(p, q, u, \frac{d q}{d p}\right), \frac{\ddot{y}}{\dot{z}^{2}}-\frac{\dot{y} z}{\dot{z}^{3}}=h\left(p, q, u, \frac{d q}{d p}, \frac{d u}{d p}\right)\end{array}$ \\
\hline$A_{3,2}$ & 4 & $\begin{array}{l}p=z, q=e^{y} \dot{y}, u=\frac{\dot{x}}{\dot{y}}, v=\frac{\dot{x}}{\dot{z}}, \frac{d q}{d p}=e^{y}\left(\frac{\ddot{y}}{\dot{z}}+\frac{\dot{y}^{2}}{\dot{z}}\right), \frac{d u}{d q}=\frac{\ddot{x}}{\dot{y}^{2}}-\frac{\dot{x} \ddot{y}}{\dot{z} \dot{y}^{2}}, \frac{d v}{d p}=\frac{\ddot{x}}{\dot{z}^{2}}-\frac{\dot{x} \ddot{z}}{\dot{z}^{3}} \\
e^{y}\left(\frac{\ddot{y}}{\dot{z}}+\frac{\dot{y}^{2}}{\dot{z}}\right)=f(p, q, u, v), \frac{\ddot{x}}{\dot{y}^{2}}-\frac{\dot{x} \ddot{y}}{\dot{z} \dot{y}^{2}}=g(p, q, u, v), \frac{\ddot{x}}{\dot{z}^{2}}-\frac{\dot{x} \ddot{z}}{\dot{z}^{3}}=h(p, q, u, v) \\
p=z, q=y, u=e^{\dot{x} \dot{y}} \dot{y}, \frac{d q}{d p}=\frac{\dot{y}}{\dot{z}}, w=\frac{\ddot{y}}{\dot{y}^{2}}, \frac{d u}{d p}=e^{\dot{x} / \dot{y}}\left(\frac{\ddot{y}}{\dot{z}}+\frac{(\dot{y}-\dot{x}) \ddot{y}}{\dot{y} \dot{z}}\right), \frac{d^{2} q}{d p^{2}}=\frac{\ddot{z}}{\dot{z} \dot{y}}-\frac{\ddot{y}}{\dot{y}^{2}} \\
e^{\dot{x} / \dot{y}}\left(\frac{\ddot{y}}{\dot{z}}+\frac{(\dot{y}-\dot{x}) \ddot{y}}{\dot{y} \dot{z}}\right)=f\left(p, q, u, \frac{d q}{d p}\right), \frac{\ddot{y}}{\dot{y}^{2}}=g\left(p, q, u, \frac{d q}{d p}\right), \frac{\ddot{z}}{\dot{z} \dot{y}}-\frac{\ddot{y}}{\dot{y}^{2}}=h\left(p, q, u, \frac{d q}{d p}\right) \\
p=z, q=y, u=\frac{\dot{x}}{\dot{y}}, \frac{d q}{d p}=\frac{\dot{y}}{\dot{z}}, w=\frac{\ddot{x}}{\dot{x}^{2}}, \frac{d u}{d p}=\frac{\ddot{x}}{\dot{y} \dot{z}}-\frac{\dot{x} \ddot{y}}{\dot{y}^{2} \dot{z}}, \frac{d^{2} q}{d p^{2}}=\frac{\ddot{y}}{\dot{z}^{2}}-\frac{\dot{y} \ddot{z}}{\dot{z}^{3}} \\
\frac{\dot{x}}{\dot{x}^{2}}=f\left(p, q, u, \frac{d q}{d p}\right), \frac{\dot{x}}{\dot{y} \dot{z}}-\frac{\dot{x} \ddot{y}}{\dot{y}^{2} \dot{z}}=g\left(p, q, u, \frac{\dot{y}}{d p}\right), \frac{\dot{y}}{\dot{z}^{2}}-\frac{\dot{y}}{\dot{z}^{3}}=h\left(p, q, u, \frac{d q}{d p}\right) \\
p=z, q=y, u=t \dot{z}, \frac{d q}{d p}=\frac{\dot{y}}{\dot{z}}, w=t \ddot{x}, \frac{d^{2} q}{d p^{2}}=\frac{\ddot{y}}{\dot{z}^{2}}-\frac{\dot{y} \ddot{z}}{\dot{z}^{3}}, \frac{d u}{d p}=1+\frac{t \ddot{z}}{\dot{z}} \\
t \ddot{x}=f\left(p, q, u, \frac{d q}{d p}\right), \frac{\ddot{y}}{\dot{z}^{2}}-\frac{\dot{y} \ddot{z}}{\dot{z}^{3}}=g\left(p, q, u, \frac{d q}{d p}\right), 1+\frac{t \ddot{z}}{\dot{z}}=h\left(p, q, u, \frac{d q}{d p}\right)\end{array}$ \\
\hline$A_{3,3}$ & 2 & $\begin{array}{l}p=z, q=y, u=\frac{\dot{x}}{\dot{y}}, \frac{d q}{d p}=\frac{\dot{y}}{\dot{z}}, w=\frac{\ddot{x}}{\dot{x}^{3}}, \frac{d u}{d p}=\frac{\ddot{x}}{\dot{y} \dot{z}}-\frac{\dot{x} \ddot{y}}{\dot{y}^{2} \dot{z}}, \frac{d^{2} q}{d p^{2}}=\frac{\ddot{y}}{\dot{z}^{2}}-\frac{\dot{y} \ddot{z}}{\dot{z}^{3}} \\
\frac{\ddot{x}}{\dot{x}^{3}}=f\left(p, q, u, \frac{d q}{d p}\right), \frac{\ddot{x}}{\dot{y} \dot{z}}-\frac{\dot{x} \ddot{y}}{\dot{y}^{2} \dot{z}}=g\left(p, q, u, \frac{d q}{d p}\right), \frac{\ddot{y}}{\dot{z}^{2}}-\frac{\dot{y} \dot{z}}{\dot{z}^{3}}=h\left(p, q, u, \frac{d q}{d p}\right) \\
p=z, q=y, u=\frac{\dot{x}^{2}}{2 \dot{y}^{2}}-\frac{1}{\dot{y}}, \frac{d q}{d p}=\frac{\dot{y}}{\dot{z}}, w=\frac{\ddot{x}}{\dot{y}^{2}}-\frac{\dot{x} \ddot{y}}{\dot{y}^{3}}, \frac{d u}{d p}=\frac{\dot{x} \ddot{x}}{\dot{y}^{2} \dot{z}}+\frac{\left(\dot{y}-\dot{x}^{2}\right) \ddot{y}}{\dot{y}^{3} \dot{z}}, \frac{d^{2} q}{d p^{2}}=\frac{\ddot{y}}{\dot{y} \dot{z}}-\frac{\ddot{z}}{\dot{z}^{2}} \\
\frac{\ddot{x}}{\dot{y}^{2}}-\frac{\dot{x} \ddot{y}}{\dot{y}^{3}}=f\left(p, q, u, \frac{d q}{d p}\right), \frac{\dot{x} \ddot{x}}{\dot{y}^{2} \dot{z}}+\frac{\left(\dot{y}-\dot{x}^{2}\right) \ddot{y}}{\dot{y}^{3} \dot{z}}=g\left(p, q, u, \frac{d q}{d p}\right), \frac{\ddot{z}}{\dot{y} \dot{z}}-\frac{\ddot{y}}{\dot{y}^{2}}=h\left(p, q, u, \frac{d q}{d p}\right) \\
p=z, q=y-\frac{1}{\dot{x}}, u=\frac{\dot{x}}{\dot{y}}, v=\frac{\dot{x}}{\dot{z}}, \frac{d q}{d p}=\frac{\ddot{x}}{\dot{x}^{2} \dot{z}}+\frac{\dot{y}}{\dot{z}}, \frac{d u}{d p}=\frac{\ddot{x}}{\dot{y} \dot{z}}-\frac{\dot{x} \ddot{y}}{\dot{y}^{2} \dot{z}}, \frac{d v}{d p}=\frac{\ddot{x}}{\dot{z}^{2}}-\frac{\dot{x} \ddot{z}}{\dot{z}^{3}} \\
\frac{\ddot{x}}{\dot{x}^{2} \dot{z}}+\frac{\dot{y}}{\dot{z}}=f(p, q, u, v), \frac{\ddot{x}}{\dot{y} \dot{z}}-\frac{\dot{x} \ddot{y}}{\dot{y}^{2} \dot{z}}=g(p, q, u, v), \frac{\ddot{x}}{\dot{z}^{2}}-\frac{\dot{x} \ddot{z}}{\dot{z}^{3}}=h(p, q, u, v)\end{array}$ \\
\hline$A_{3,4}$ & 1 & $\begin{array}{l}p=z, q=y, u=e^{1 / \dot{x}} \frac{\dot{y}}{\dot{x}}, \frac{d q}{d p}=\frac{\dot{y}}{\dot{z}}, w=\frac{\ddot{x}}{\dot{x}^{2} \dot{y}}, \frac{d u}{d p}=e^{1 / \dot{x}}\left(\frac{\ddot{y}}{\dot{x} \dot{z}}-\frac{(1+\dot{x}) \ddot{x} \dot{y}}{\dot{x}^{3} \dot{z}}\right), \frac{d^{2} q}{d p^{2}}=\frac{\ddot{y}}{\dot{z}^{2}}-\frac{\dot{y} \ddot{y}}{\dot{z}^{3}} \\
\frac{\ddot{x}}{\dot{x}^{2} \dot{y}}=f\left(p, q, u, \frac{d q}{d p}\right), e^{1 / \dot{x}}\left(\frac{\ddot{y}}{\dot{x} \dot{z}}-\frac{(1+\dot{x}) \ddot{x} \dot{y}}{\dot{x}^{3} \dot{z}}\right)=g\left(p, q, u, \frac{d q}{d p}\right), \frac{\ddot{y}}{\dot{z}^{2}}-\frac{\dot{y} \ddot{y}}{\dot{z}^{3}}=h\left(p, q, u, \frac{d q}{d p}\right) \\
p=z, q=y-\frac{1}{\dot{x}}, u=e^{1 / \dot{x}} \frac{\dot{y}}{\dot{x}}, v=e^{1 / \dot{x}} \frac{\dot{z}}{\dot{x}}, \frac{d q}{d p}=\frac{\dot{y}}{\dot{z}}\left(1+\frac{\ddot{x}}{\dot{x}^{2} \dot{y}}\right) \\
\frac{d u}{d p}=e^{1 / \dot{x}}\left(\frac{\ddot{y}}{\dot{x} \dot{z}}-\frac{(1+\dot{x}) \ddot{x} \dot{y}}{\dot{x}^{3} \dot{z}}\right), \frac{d v}{d p}=e^{1 / \dot{x}}\left(\frac{\dot{z}}{\dot{x} \dot{z}}-\frac{(1+\dot{x}) \ddot{x}}{\dot{x}^{3}}\right) \\
\frac{\dot{y}}{\dot{z}}\left(1+\frac{\ddot{x}}{\dot{x}^{2} \dot{y}}\right)=f(p, q, u, v), e^{1 / \dot{x}}\left(\frac{\ddot{y}}{\dot{x} \dot{z}}-\frac{(1+\dot{x}) \ddot{x} \dot{y}}{\dot{x}^{3} \dot{z}}\right)=g(p, q, u, v), e^{1 / \dot{x}}\left(\frac{\ddot{z}}{\dot{x} \dot{z}}-\frac{(1+\dot{x}) \ddot{x}}{\dot{x}^{3}}\right)=h(p, q, u, v) \\
p=z, q=y, u=\dot{z}, \frac{d q}{d p}=\frac{\dot{y}}{\dot{z}}, w=e^{t} \ddot{x}, \frac{d u}{d p}=\frac{\ddot{z}}{\dot{z}}, \frac{d^{2} q}{d p^{2}}=\frac{\ddot{y}}{\dot{z}^{2}}-\frac{\dot{y} \ddot{z}}{\dot{z}^{3}} \\
e^{t} \ddot{x}=f\left(p, q, u, \frac{d q}{d p}\right), \frac{\ddot{y}}{\dot{z}^{2}}-\frac{\dot{y} \ddot{z}}{\dot{z}^{3}}=g\left(p, q, u, \frac{d q}{d p}\right), \frac{\ddot{z}}{\dot{z}}=h\left(p, q, u, \frac{d q}{d p}\right)\end{array}$ \\
\hline
\end{tabular}


TABLe 1: Continued.

\begin{tabular}{|c|c|c|}
\hline Algebra & $N$ & Invariants and equations \\
\hline$A_{3,5}$ & 3 & $\begin{array}{l}p=z, q=y, u=\dot{x}, \frac{d q}{d p}=\frac{\dot{y}}{\dot{z}}, w=\frac{\ddot{y}}{\dot{y}^{2}}, \frac{d u}{d p}=\frac{\ddot{x}}{\dot{z}}, \frac{d^{2} q}{d p^{2}}=\frac{\dot{y} \ddot{z}}{\dot{z}^{3}}-\frac{\ddot{y}}{\dot{z}^{2}} \\
\frac{\ddot{x}}{\dot{z}}=f\left(p, q, u, \frac{d q}{d p}\right), \frac{\ddot{y}}{\dot{y}^{2}}=g\left(p, q, u, \frac{d q}{d p}\right), \frac{\dot{y} \ddot{z}}{\dot{z}^{3}}-\frac{\ddot{y}}{\dot{z}^{2}}=h\left(p, q, u, \frac{d q}{d p}\right) \\
p=z, q=\dot{x}, u=e^{y} \dot{y}, v=\frac{\dot{z}}{\dot{y}}, \frac{d q}{d p}=\frac{\ddot{x}}{\dot{z}}, \frac{d u}{d q}=e^{y} \frac{\dot{y}^{2}}{\dot{z}}+e^{y} \frac{\ddot{y}}{\dot{y} \dot{z}}, \frac{d v}{d p}=\frac{\ddot{z}}{\dot{y} \dot{z}}-\frac{\ddot{y}}{\dot{y}^{2}} \\
\frac{\ddot{x}}{\dot{z}}=f(p, q, u, v), e^{y} \frac{\dot{y}^{2}}{\dot{z}}+e^{y} \frac{\ddot{y}}{\dot{z}}=g(p, q, u, v), \frac{\ddot{z}}{\dot{y} \dot{z}}-\frac{\ddot{y}}{\dot{y}^{2}}=h(p, q, u, v) \\
p=t, q=y, u=z, \frac{d q}{d p}=\dot{z}, \frac{d u}{d p}=\dot{z}, \frac{d^{2} q}{d p^{2}}=\ddot{y}, w=\ddot{x}=0, \frac{d^{2} u}{d p^{2}}=\ddot{z} \\
\ddot{x}=0, \ddot{y}=g\left(p, q, u, \frac{d q}{d p}, \frac{d u}{d p}\right), \ddot{z}=h\left(p, q, u, \frac{d q}{d p}, \frac{d u}{d p}\right) \\
p=t, q=z, u=\dot{y}, \frac{d q}{d p}=\dot{z}, w=e^{-y} \ddot{x}, \frac{d u}{d p}=\ddot{y}, \frac{d^{2} q}{d p^{2}}=\ddot{z} \\
e^{-y} \ddot{x}=f\left(p, q, u, \frac{d q}{d p}\right), \ddot{y}=g\left(p, q, u, \frac{d q}{d p}\right), \frac{\ddot{z}}{\dot{z}}=h\left(p, q, u, \frac{d q}{d p}\right)\end{array}$ \\
\hline $\begin{array}{l}A_{3,6}^{a} \\
|a| \leq 1 \\
a \neq 0,1\end{array}$ & 2 & $\begin{array}{l}p=z, q=y, u=\dot{x} \dot{y}^{a-1}, \frac{d q}{d p}=\frac{\dot{y}}{\dot{z}}, w=\frac{\ddot{x}}{\dot{x} \dot{y}}, \frac{d u}{d p}=\frac{\dot{y}^{(a-1)} \ddot{x}}{\dot{z}}+\frac{(a-1) \dot{x} \dot{y}^{(a-2)} \ddot{y}}{\dot{z}}, \frac{d^{2} q}{d p^{2}}=\frac{\ddot{y}}{\dot{y} \dot{z}^{2}}-\frac{\dot{y} \ddot{z}}{\dot{z}^{3}} \\
\frac{\ddot{x}}{\dot{x} \dot{y}}=f\left(p, q, u, \frac{d q}{d p}\right), \frac{\dot{y}^{(a-1)} \ddot{x}}{\dot{z}}+\frac{(a-1) \dot{x} \dot{y}^{(a-2)} \ddot{y}}{\dot{z}}=g\left(p, q, u, \frac{d q}{d p}\right), \frac{\ddot{y}}{\dot{y} \dot{z}^{2}}-\frac{\dot{y} \ddot{z}}{\dot{z}^{3}}=h\left(p, q, u, \frac{d q}{d p}\right) \\
p=z, q=e^{y} \dot{y}, u=\dot{x} \dot{y}^{a-1}, v=\frac{\dot{z}}{\dot{y}}, \frac{d q}{d p}=e^{y}\left(\frac{\ddot{y}}{\dot{z}}+\frac{\dot{y}^{2}}{\dot{z}}\right), \frac{d u}{d q}=\frac{\dot{y}^{(a-1)} \ddot{x}}{\dot{z}}+\frac{(a-1) \dot{x} \dot{y}^{(a-2)} \ddot{y}}{\dot{z}}, \frac{d v}{d p}=\frac{\ddot{z}}{\dot{y} \dot{z}}-\frac{\ddot{y}}{\dot{y}^{2}} \\
e^{y}\left(\frac{\ddot{y}}{\dot{z}}+\frac{\dot{y}^{2}}{\dot{z}}\right)=f(p, q, u, v), \frac{\dot{y}^{(a-1)} \ddot{x}}{\dot{z}}+\frac{(a-1) \dot{x} \dot{y}^{(a-2)} \ddot{y}}{\dot{z}}=g(p, q, u, v), \frac{\ddot{z}}{\dot{y} \dot{z}}-\frac{\ddot{y}}{\dot{y}^{2}}=h(p, q, u, v) \\
p=z, q=y, u=t \dot{z}, \frac{d q}{d p}=\frac{\dot{y}}{\dot{z}}, w=t^{(2 a-1) /(a-1)} \ddot{x}, \frac{d u}{d p}=1+\frac{t \ddot{z}}{\dot{z}}, \frac{d^{2} q}{d p^{2}}=\frac{\ddot{y}}{\dot{z}^{2}}-\frac{\dot{y} \ddot{z}}{\dot{z}^{3}} \\
t^{(2 a-1) /(a-1)} \ddot{x}=f\left(p, q, u, \frac{d q}{d p}\right), \frac{\ddot{y}}{\dot{z}^{2}}-\frac{\dot{y} \ddot{z}}{\dot{z}^{3}}=g\left(p, q, u, \frac{d q}{d p}\right), 1+\frac{t \ddot{z}}{\dot{z}}=h\left(p, q, u, \frac{d q}{d p}\right)\end{array}$ \\
\hline$A_{3,7}$ & 2 & $\begin{array}{l}p=z, q=y, u=\frac{\dot{y} e^{-a \arctan \dot{x}}}{\sqrt{1+\dot{x}^{2}}}, v=\frac{\dot{y}}{\dot{z}}, w=\frac{\ddot{x} e^{-a \arctan \dot{x}}}{\left(1+\dot{x}^{2}\right)^{3 / 2}}, \frac{d u}{d p}=\frac{e^{-a \arctan \dot{x}}}{\sqrt{1+\dot{x}^{2}}}\left(\frac{\ddot{y}}{\dot{z}}-\frac{(a+\dot{x}) \dot{y} \ddot{x}}{\dot{z}\left(1+\dot{x}^{2}\right)}\right), \frac{d^{2} q}{d p^{2}}=\frac{\ddot{y}}{\dot{z}^{2}}-\frac{\dot{y} \ddot{z}}{\dot{z}^{3}} \\
\frac{\ddot{x} e^{-a \arctan \dot{x}}}{\left(1+\dot{x}^{2}\right)^{3 / 2}}=f\left(p, q, u, \frac{d q}{d p}\right), \frac{e^{-a \arctan \dot{x}}}{\sqrt{1+\dot{x}^{2}}}\left(\frac{\ddot{y}}{\dot{z}}-\frac{(a+\dot{x}) \dot{y} \ddot{x}}{\dot{z}\left(1+\dot{x}^{2}\right)}\right)=g\left(p, q, u, \frac{d q}{d p}\right), \frac{\ddot{y}}{\dot{z}^{2}}-\frac{\dot{y} \ddot{z}}{\dot{z}^{3}}=h\left(p, q, u, \frac{d q}{d p}\right) \\
p=z, q=y+\arctan \dot{x}, u=\frac{\dot{y} e^{-a \arctan \dot{x}}}{\sqrt{1+\dot{x}^{2}}}, v=\frac{\dot{z} e^{-a \arctan \dot{x}}}{\sqrt{1+\dot{x}^{2}}}, \frac{d q}{d p}=\frac{\dot{y}}{\dot{z}}+\frac{\ddot{x}}{\dot{z}\left(1+\dot{x}^{2}\right)} \\
\frac{d u}{d p}=\frac{e^{-a \arctan \dot{x}}}{\sqrt{1+\dot{x}^{2}}}\left(\frac{\ddot{y}}{\dot{z}}-\frac{(a+\dot{x})}{\dot{y} \ddot{x}}\right), \frac{d v}{d p}=\frac{e^{-a \arctan \dot{x}}}{\sqrt{1+\dot{x}^{2}}}\left(\frac{\ddot{z}}{\dot{z}}-\frac{(a+\dot{x}) \ddot{x}}{\left(1+\dot{x}^{2}\right)}\right) \\
\frac{\dot{y}}{\dot{z}}+\frac{\ddot{x}}{\dot{z}\left(1+\dot{x}^{2}\right)}=f(p, q, u, v), \frac{e^{-a \arctan \dot{x}}}{\sqrt{1+\dot{x}^{2}}}\left(\frac{\ddot{y}}{\dot{z}}-\frac{(a+\dot{x}) \dot{y} \ddot{x}}{\dot{z}\left(1+\dot{x}^{2}\right)}\right)=g(p, q, u, v) \\
\frac{e^{-a \arctan \dot{x}}}{\sqrt{1+\dot{x}^{2}}}\left(\frac{\ddot{z}}{\dot{z}}-\frac{(a+\dot{x}) \ddot{x}}{\left(1+\dot{x}^{2}\right)}\right)=h(p, q, u, v) \\
p=z, q=y, u=\left(1+t^{2}\right) \dot{z}, \frac{d q}{d p}=\frac{\dot{y}}{\dot{z}}, w=\frac{\ddot{x} e^{a \arctan t}}{\dot{y}^{3 / 2}}, \frac{d u}{d p}=2 t+\frac{\left(1+t^{2}\right) \ddot{z}}{\dot{z}}, \frac{d^{2} q}{d p^{2}}=\frac{\ddot{y}}{\dot{z}^{2}}-\frac{\dot{y} \ddot{z}}{\dot{z}^{3}} \\
\frac{\ddot{x} e^{a \arctan t}}{\dot{y}^{3 / 2}}=f\left(p, q, u, \frac{d q}{d p}\right), \frac{\ddot{y}}{\dot{z}^{2}}-\frac{\dot{y} \ddot{z}}{\dot{z}^{3}}=g\left(p, q, u, \frac{d q}{d p}\right), 2 t+\frac{\left(1+t^{2}\right) \ddot{z}}{\dot{z}}=h\left(p, q, u, \frac{d q}{d p}\right)\end{array}$ \\
\hline$A_{3,8}$ & 2 & $\begin{array}{l}p=z, q=2 y-\dot{x}, u=\dot{x}^{2}-4 x \dot{y}, v=x \dot{z}, \frac{d q}{d p}=\frac{(2 \dot{y}-\ddot{x})}{\dot{z}}, \frac{d u}{d p}=\frac{2 \dot{x} \ddot{x}-4 x \ddot{y}-4 \dot{x} \ddot{y}}{\dot{z}}, \frac{d v}{d p}=\dot{x}+\frac{x \ddot{z}}{\dot{z}} \\
\frac{(2 \dot{y}-\ddot{x})}{\dot{z}}=f(p, q, u, v), \frac{2 \dot{x} \ddot{x}-4 x \ddot{y}-4 \dot{x} \ddot{y}}{\dot{z}}=g(p, q, u, v), \dot{x}+\frac{x \ddot{z}}{\dot{z}}=h(p, q, u, v) \\
p=z, q=y, u=\frac{\dot{x}}{(t-x)^{2} \dot{y}^{2}}, v=\frac{\dot{y}}{\dot{z}}, w=\frac{2}{\dot{x}^{1 / 2}}+\frac{(t-x) \ddot{x}}{\dot{x}^{3 / 2}}+2 \sqrt{x} \\
\frac{d u}{d p}=\frac{\ddot{x}}{(t-x)^{2} \dot{y}^{2} \dot{z}}-\frac{2(1-\dot{x}) \dot{x} \dot{y}+2(t-x) \dot{x} \ddot{y}}{(t-x)^{3} \dot{y}^{3} \dot{z}}, \frac{d^{2} q}{d q^{2}}=\frac{\ddot{y}}{\dot{z}^{2}}-\frac{\dot{y} \ddot{z}}{\dot{z}^{3}} \\
\frac{2}{\dot{x}^{1 / 2}}+\frac{(t-x) \ddot{x}}{\dot{x}^{3 / 2}}+2 \sqrt{x}=f\left(p, q, u, \frac{d q}{d p}\right), \frac{\dot{x}}{(t-x)^{2} \dot{y}^{2} \dot{z}}-\frac{2(1-\dot{x}) \dot{x} \dot{y}+2(t-x) \dot{x} \ddot{y}}{(t-x)^{3} \dot{y}^{3} \dot{z}}=g\left(p, q, u, \frac{d q}{d p}\right), \\
\frac{\ddot{y}}{\dot{z}^{2}}-\frac{\dot{y} \ddot{z}}{\dot{z}^{3}}=h\left(p, q, u, \frac{d q}{d p}\right)\end{array}$ \\
\hline
\end{tabular}


TABLE 1: Continued.

\begin{tabular}{|c|c|c|}
\hline Algebra & $N$ & Invariants and equations \\
\hline & 5 & $\begin{array}{l}p=z, q=y, u=\frac{t \dot{x}-x}{\dot{y}}, v=\frac{\dot{y}}{\dot{z}}, w=\frac{\ddot{x}}{\dot{y}^{3}}, \frac{d u}{d p}=\frac{t \ddot{x}}{\dot{y} \dot{z}}-\frac{(t \dot{x}-x) \ddot{y}}{\dot{z} \dot{y}^{2}}, \frac{d^{2} q}{d p^{2}}=\frac{\ddot{y}}{\dot{z}^{2}}-\frac{\dot{y} \ddot{z}}{\dot{z}^{3}} \\
\frac{\ddot{x}}{\dot{y}^{3}}=f\left(p, q, u, \frac{d q}{d p}\right), \frac{t \ddot{x}}{\dot{y} \dot{z}}-\frac{(t \dot{x}-x) \ddot{y}}{\dot{z} \dot{y}^{2}}=g\left(p, q, u, \frac{d q}{d p}\right), \frac{\ddot{y}}{\dot{z}^{2}}-\frac{\dot{y} \ddot{z}}{\dot{z}^{3}}=h\left(p, q, u, \frac{d q}{d p}\right) \\
p=z, q=y, u=\frac{x \dot{z}}{\sqrt{1+\dot{x}^{2}}}, \frac{d q}{d p}=\frac{\dot{y}}{\dot{z}}, w=\frac{1+\dot{x}^{2}}{x^{3} \dot{y}^{3}}+\frac{\ddot{x}}{x^{2} \dot{y}^{3}}, \frac{d u}{d p}=\frac{\sqrt{1+\dot{x}^{2}}}{x \dot{z}}\left(\frac{x \dot{x} \dot{z}}{\left(1+\dot{x}^{2}\right)}+\frac{x^{2} \ddot{z}}{\sqrt{1+\dot{x}^{2}}}-\frac{x^{2} \dot{x} \dot{z} \ddot{x}}{\left(1+\dot{x}^{2}\right)}\right) \\
\frac{d^{2} q}{d p^{2}}=\frac{\ddot{y}}{\dot{z}^{2}}-\frac{\dot{y} \ddot{z}}{\dot{z}^{3}} \\
\frac{1+\dot{x}^{2}}{x^{3} \dot{y}^{3}}+\frac{\ddot{x}}{x^{2} \dot{y}^{3}}=f\left(p, q, u, \frac{d q}{d p}\right), \frac{\ddot{y}}{\dot{z}^{2}}-\frac{\dot{y} \ddot{z}}{\dot{z}^{3}}=g\left(p, q, u, \frac{d q}{d p}\right) \\
\frac{\sqrt{1+\dot{x}^{2}}}{x \dot{z}}\left(\frac{x \dot{x} \dot{z}}{\left(1+\dot{x}^{2}\right)}+\frac{x^{2} \ddot{z}}{1+\dot{x}^{2}}-\frac{x^{2} \dot{x} \dot{z} \ddot{x}}{\left(1+\dot{x}^{2}\right)}\right)=h\left(p, q, u, \frac{d q}{d p}\right)\end{array}$ \\
\hline$A_{3,9}$ & 2 & 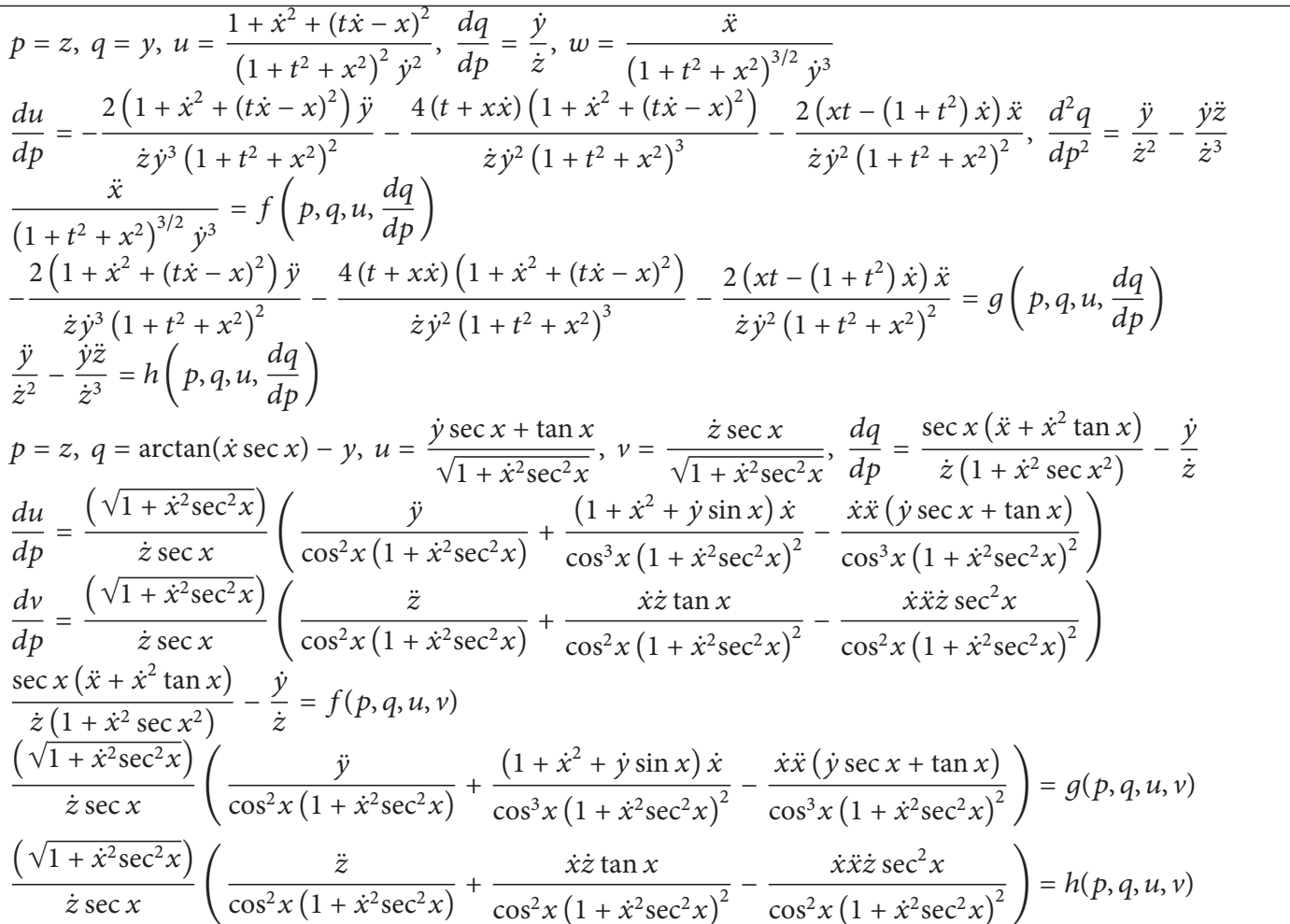 \\
\hline
\end{tabular}

$$
\begin{aligned}
& \frac{d w}{d p}, \\
& \frac{d^{2} u}{d p^{2}}, \\
& \frac{d^{3} q}{d p^{3}},
\end{aligned}
$$

where $d w / d p, d^{2} u / d p^{2}, d^{3} q / d p^{3}$ are as mentioned in (9). Consequently, the number of functionally independent invariants for the system of three 3rd-order ODEs admitting $\mathscr{A}_{3,8}^{4}$ is 10 . We arrive at the following invariant representation as well as corresponding canonical form for the system of three 3rd-order ODEs possessing Lie algebra $\mathscr{A}_{3,8}^{4}$ :

$$
\begin{aligned}
& \frac{d w}{d p}=f\left(p, q, u, \frac{d q}{d p}, w, \frac{d u}{d p}, \frac{d^{2} q}{d p^{2}}\right) \\
& \frac{d^{2} u}{d p^{2}}=g\left(p, q, u, \frac{d q}{d p}, w, \frac{d u}{d p}, \frac{d^{2} q}{d p^{2}}\right) \\
& \frac{d^{3} q}{d p^{3}}=h\left(p, q, u, \frac{d q}{d p}, w, \frac{d u}{d p}, \frac{d^{2} q}{d p^{2}}\right) \\
& \frac{\dddot{x}}{\dot{z} \dot{y}^{3}}-3 \frac{\ddot{x} \ddot{y}}{\dot{z} \dot{y}^{4}}=f\left(p, q, u, \frac{d q}{d p}, w, \frac{d u}{d p}, \frac{d^{2} q}{d p^{2}}\right) \\
& \frac{\dot{z}(t \ddot{x}+\dot{x})-t \dot{x} \ddot{z}}{\dot{y} \dot{z}^{3}}-\frac{\ddot{y}(t \ddot{x}+\dot{x}(t-1))}{\dot{y}^{2} \dot{z}^{2}} \\
& +\frac{\ddot{y}(t \dot{x}-x)(2 \dot{z} \ddot{y}+\dot{y} \ddot{z})}{\dot{y}^{3} \dot{z}^{3}}
\end{aligned}
$$




$$
\begin{gathered}
-\frac{\dddot{y}(t \dot{x}-x)}{\dot{y}^{2} \dot{z}^{2}}=g\left(p, q, u, \frac{d q}{d p}, w, \frac{d u}{d p}, \frac{d^{2} q}{d p^{2}}\right) \\
\frac{\dddot{y}}{\dot{z}^{3}}-3 \frac{\ddot{y} \ddot{z}}{\dot{z}^{4}}+3 \frac{\dot{y} \ddot{z}^{2}}{\dot{z}^{5}} \\
-\frac{\dot{y} \dddot{z}}{\dot{z}^{3}}=h\left(p, q, u, \frac{d q}{d p}, w, \frac{d u}{d p}, \frac{d^{2} q}{d p^{2}}\right) .
\end{gathered}
$$

Proceeding in similar manner, we arrive at the following invariant representation for the system of three $k$ th-order ODEs $(k \geq 3)$ possessing Lie algebra $\mathscr{A}_{3,8}^{4}$ :

$$
\begin{aligned}
& \frac{d^{k-2} w}{d p^{k-2}}=f\left(p, q, u, \frac{d q}{d p}, w, \frac{d u}{d p}, \frac{d^{2} q}{d p^{2}}, \ldots, \frac{d^{k-3} w}{d p^{k-3}}, \frac{d^{k-2} u}{d p^{k-2}},\right. \\
& \left.\frac{d^{k-1} q}{d p^{k-1}}\right)
\end{aligned}
$$

$$
\begin{aligned}
& \frac{d^{k-1} u}{d p^{k-1}}=g\left(p, q, u, \frac{d q}{d p}, w, \frac{d u}{d p}, \frac{d^{2} q}{d p^{2}}, \ldots, \frac{d^{k-3} w}{d p^{k-3}}, \frac{d^{k-2} u}{d p^{k-2}},\right. \\
& \left.\frac{d^{k-1} q}{d p^{k-1}}\right) \\
& \frac{d^{k} q}{d p^{k}}=h\left(p, q, u, \frac{d q}{d p}, w, \frac{d u}{d p}, \frac{d^{2} q}{d p^{2}}, \ldots, \frac{d^{k-3} w}{d p^{k-3}}, \frac{d^{k-2} u}{d p^{k-2}},\right. \\
& \left.\frac{d^{k-1} q}{d p^{k-1}}\right) .
\end{aligned}
$$

By careful observation we can classify the invariant representation for all cases of the three-dimensional Lie algebras with corresponding realization admitted by the system of three $k$ th-order ordinary differential equations $(k \geq 3)$ admitting three-dimensional Lie algebra.

Theorem 5. If a regular system of three kth-order ordinary differential equations $(k \geq 3)$ admits three-dimensional Lie algebra then its canonical form can be represented by a set of basic $3 k+1$ functionally independent invariants in one of the following forms:

$$
\begin{aligned}
& \text { (a) } p, q, u, v, \frac{d q}{d p}, \frac{d u}{d p}, \frac{d v}{d p}, \frac{d^{2} q}{d p^{2}}, \frac{d^{2} u}{d p^{2}}, \frac{d^{2} v}{d p^{2}}, \ldots, \frac{d^{k-1} q}{d p^{k-1}}, \frac{d^{k-1} u}{d p^{k-1}}, \frac{d^{k-1} v}{d p^{k-1}} \\
& \text { (b) } p, q, u, \frac{d q}{d p}, w, \frac{d u}{d p}, \frac{d^{2} q}{d p^{2}}, \frac{d w}{d p}, \frac{d^{2} u}{d p^{2}}, \frac{d^{3} u}{d p^{3}}, \ldots, \frac{d^{k-2} w}{d p^{k-2}}, \frac{d^{k-1} u}{d p^{k-1}}, \frac{d^{k} q}{d p^{k}} \\
& \text { (c) } p, q, u, \frac{d q}{d p}, \frac{d u}{d p}, \frac{d^{2} q}{d p^{2}}, \frac{d^{2} u}{d p^{2}}, w, \frac{d^{3} q}{d p^{3}}, \frac{d^{3} u}{d p^{3}}, \ldots, \frac{d^{k-3} w}{d p^{k-3}}, \frac{d^{k} q}{d p^{k}}, \frac{d^{k} u}{d p^{k}}
\end{aligned}
$$

where (a) corresponds to these algebras

$$
A_{3,1}^{1}, A_{3,2}^{1}, A_{3,2}^{2}, A_{3,3}^{3}, A_{3,5}^{2}, A_{3,6}^{2}, A_{3,7}^{2}, A_{3,8}^{2}, A_{3,9}^{2} \text {, }
$$

(b) corresponds to

$$
\begin{gathered}
A_{3,1}^{2}, A_{3,2}^{2}, A_{3,2}^{3}, A_{3,2}^{4}, A_{3,3}^{1}, A_{3,3}^{2}, A_{3,4}^{1}, A_{3,4}^{3}, A_{3,5}^{1}, A_{3,5}^{4}, A_{3,6}^{1}, \\
A_{3,6}^{3}, A_{3,7}^{1}, A_{3,7}^{3}, A_{3,8}^{3}, A_{3,8}^{4}, A_{3,8}^{5}, A_{3,9}^{1},
\end{gathered}
$$

and (c) corresponds to

$$
A_{3,1}^{3}, A_{3,1}^{4}, A_{3,5}^{3}, A_{3,8}^{1} .
$$

Remarks 6. The classification results of (a) and (b) mentioned in Theorem 5 are also valid for $k=2$.

There are 31 canonical forms for the case of a system of three 3rd- and higher-order ordinary differential equations admitting three-dimensional Lie algebras, and these can be constructed by invariant differentiation as described above.

Note that $A_{3,1}^{4}$ and $A_{3,8}^{1}$ are admitted by a regular system of two 3rd- and higher-order ordinary differential equations whereas they do not form a system of three 2nd-order ordinary differential equations.

\section{Integrability}

It is well known that symmetries can be used to integrate ODEs either by successive reduction or by using the canonical forms. Though the later method requires classification according to their admitted algebras before integration but this method is applicable to all kinds of systems of ODEs whereas the former applies only to scalar ODEs. Here we provide an integration algorithm for canonical forms (given in Table 1). The algorithm depends on the subdivision scheme of the canonical forms that is based on general observations.

3.1. Integration Algorithm. The canonical forms for systems of three 2 nd-order ODEs presented in Table 1 are subdivided mainly in two classes: Type-I and Type-II.

Type-I: Case of Singular Invariants. This consists of two cases $A_{3,1}^{3}$ and $A_{3,5}^{3}$.

(i) $A_{3,1}^{3} \simeq\left\langle\partial_{t}, \partial_{x}, x \partial_{t}+\partial_{y}\right\rangle$. The corresponding canonical form and invariant representation for this symmetry Lie algebra are

$$
\ddot{x}=f\left(p, q, u, \frac{d q}{d p}, \frac{d u}{d p}\right),
$$




$$
\begin{aligned}
\ddot{y} & =0, \\
\ddot{z} & =h\left(p, q, u, \frac{d q}{d p}, \frac{d u}{d p}\right), \\
w & =f\left(p, q, u, \frac{d q}{d p}, \frac{d u}{d p}\right), \\
\frac{d^{2} q}{d p^{2}} & =0, \\
\frac{d^{2} u}{d p^{2}} & =h\left(p, q, u, \frac{d q}{d p}, \frac{d u}{d p}\right),
\end{aligned}
$$

where $p, q, u, d q / d p$ and $d u / d p$ are taken from Table 1. By using (21), we arrive at

$$
q=c_{1} p+c_{2}
$$

Thus utilizing the values of (23) in (20) and (22), we deduce that

$$
\begin{aligned}
w & =f\left(p, c_{1} p+c_{2}, u, c_{1}, \frac{d u}{d p}\right) \\
\frac{d^{2} u}{d p^{2}} & =h\left(p, c_{1} p+c_{2}, u, c_{1}, \frac{d u}{d p}\right) .
\end{aligned}
$$

Inverting back into original variables, (23) and (24), we get the following form:

$$
\begin{aligned}
& y=c_{1} t+c_{2} \\
& \ddot{x}=f\left(t, c_{1} t+c_{2}, z, c_{1}, \frac{d z}{d t}\right) \\
& \ddot{z}=h\left(t, c_{1} t+c_{2}, z, c_{1}, \frac{d z}{d t}\right) .
\end{aligned}
$$

The solution of (27) gives the value of $z$, and then by utilizing this value in (26), one can get the solution of system (17), (18), and (19).

Remark 7. The solution of (27) can be obtained by employing different symmetry approaches including Lie canonical form method. (ii) $A_{3,5}^{3} \simeq\left\langle\partial_{x}, t \partial_{x}, x \partial_{x}\right\rangle$. The associated canonical form possessing symmetry Lie algebra is

$$
\begin{aligned}
& \ddot{x}=0, \\
& \ddot{y}=g\left(p, q, u, \frac{d q}{d p}, \frac{d u}{d p}\right), \\
& \ddot{z}=h\left(p, q, u, \frac{d q}{d p}, \frac{d u}{d p}\right),
\end{aligned}
$$

where $p, q, u, d q / d p$ and $d u / d p$ are taken from Table 1. By using (28), we deduce that

$$
x=d_{1} t+d_{2} .
$$

The system of three second-order ODEs admitting symmetry Lie algebra $A_{3,5}^{3}$ is integrable by quadrature provided that system of two second-order ODEs formed by (29) and (30) is integrable by quadrature.

On the basis of reductions discussed above, we arrive at the following results.

Proposition 8. If a singular system of three 2 nd-order ordinary differential equations admits a three-dimensional solvable symmetry algebra, then the general solution of the singular system can be obtained by quadratures from the general solution of the invariant representation of the singular system given by the solution of

(a) a 2nd-order differential equation in the case of $A_{3,1}^{3}$;

(b) system of two 2nd-order ordinary differential equations in the case of $A_{3,5}^{3}$.

Remark 9. Proposition 8 is related to algebraic approach for analysis of singular system of ODEs. Proposition 8 leads to the solution of underlying singular system of three 2nd-order ODEs by either of two ways: case (a) when corresponding reduced 2nd-order differential equation has integrable class or case (b) when associated system of two 2nd-order ordinary differential equations is integrable. In both of the above cases ((a) and (b)), for integrability one can consult the Lie canonical form method of scalar second-order ODEs and other symmetry approaches as well in the former case and in latter case one can utilize approaches discussed in $[6,8,9,13]$.

Proposition 10. If a regular system of three $k$ th-order $(k \geq 3)$ ordinary differential equations possesses a three-dimensional solvable symmetry algebra, then the general solution of the system can be obtained by quadratures from the general solution of the invariant representation given by one of the following systems: (a) $p, q, u, v, \frac{d q}{d p}, \frac{d u}{d p}, \frac{d v}{d p}, \frac{d^{2} q}{d p^{2}}, \frac{d^{2} u}{d p^{2}}, \frac{d^{2} v}{d p^{2}}, \ldots, \frac{d^{k-1} q}{d p^{k-1}}, \frac{d^{k-1} u}{d p^{k-1}}, \frac{d^{k-1} v}{d p^{k-1}}$
(b) $p, q, u, \frac{d q}{d p}, w, \frac{d u}{d p}, \frac{d^{2} q}{d p^{2}}, \frac{d w}{d p}, \frac{d^{2} u}{d p^{2}}, \frac{d^{3} u}{d p^{3}}, \ldots, \frac{d^{k-2} w}{d p^{k-2}}, \frac{d^{k-1} u}{d p^{k-1}}, \frac{d^{k} q}{d p^{k}}$
(c) $p, q, u, \frac{d q}{d p}, \frac{d u}{d p}, \frac{d^{2} q}{d p^{2}}, \frac{d^{2} u}{d p^{2}}, w, \frac{d^{3} q}{d p^{3}}, \frac{d^{3} u}{d p^{3}}, \ldots, \frac{d^{k-3} w}{d p^{k-3}}, \frac{d^{k} q}{d p^{k}}, \frac{d^{k} u}{d p^{k}}$. 
The proof follows from Proposition 8 and the discussion preceding Proposition 8.

Remark 11. Proposition 10 is also related to algebraic approach for analysis of regular system of ODEs. Proposition 10 leads to the solution of underlying regular systems of three $k$ th-order $(k \geq 3)$ ODEs admitting a three-dimensional solvable symmetry algebra when corresponding canonical forms of these systems given in cases (a), (b), and (c) are integrable.

Type-II: Case of Regular Invariants. This type consists of all the cases of regular invariants mentioned in Table 1 and their integrability depends on the peculiar structure of associated system of three 2 nd-order ordinary differential equations with admitting Lie algebras.

3.1.1. Examples. Let us consider two examples of physical systems which describe the integration algorithm that depends on the peculiar structure of associated system of three 2ndorder ODEs and the admitted Lie algebra.

(1) Consider the motion of a system of three particles defined by

$$
\begin{aligned}
& \ddot{x}=-\dot{z} \\
& \ddot{y}=-\dot{y}^{2} \\
& \ddot{z}=-\dot{y} \dot{z}
\end{aligned}
$$

in a small region; $a_{1}<x<a_{2}, b_{1}<y<b_{2}$ and $c_{1}<$ $z<c_{2}$ such that for any small number $\epsilon>0,\left|a_{1}-a_{2}\right|^{2}=$ $\epsilon,\left|b_{1}-b_{2}\right|^{2}=\epsilon$ and $\left|c_{1}-c_{2}\right|^{2}=\epsilon$, where $\epsilon \rightarrow 0$. There is a time varying applied force which produces the motion after neglecting the effects of gravity due to small region. System (33) represents the motion of system of interacting particles in which the first particle faces the resistance proportional to the velocity of third particle, while second particle faces the drag resistance and third particle is facing resistance equal to the product of the velocities of second and third. This system admits the symmetry Lie algebra $A_{3,5}^{1}$ and belongs to the class of canonical form associated with this algebra with $f=g=-1$ and $h=0$. The corresponding invariants and invariant representation for $A_{3,5}^{1}$ are

$$
\begin{aligned}
p & =z, \\
q & =y, \\
u & =\dot{x}, \\
\frac{d q}{d p} & =\frac{\dot{y}}{\dot{z}}, \\
w & =\frac{\ddot{y}}{\dot{y}^{2}}, \\
\frac{d u}{d p} & =\frac{\ddot{x}}{\dot{z}}, \\
\frac{d^{2} q}{d p^{2}} & =\frac{\dot{y} \ddot{z}}{\dot{z}^{3}}-\frac{\ddot{y}}{\dot{z}^{2}} .
\end{aligned}
$$

Plugging in the values of $f, g$, and $h$ we arrive at

$$
\begin{aligned}
w & =-1 \\
\frac{d u}{d p} & =-1 \\
\frac{d^{2} q}{d p^{2}} & =0 \\
w & =-1 \Longrightarrow-\frac{\ddot{y}}{\dot{y}^{2}}=1,
\end{aligned}
$$

which gives

$$
\frac{1}{\dot{y}}=t+d_{1} \text {. }
$$

Some manipulations give

$$
y=\ln \left(t+d_{1}\right)+d_{2} .
$$

Similarly

$$
\frac{d^{2} q}{d p^{2}}=0
$$

which gives

$$
y=d_{3} z+d_{4}
$$

We deduce that

$$
z=\frac{\ln \left(t+d_{1}\right)}{d_{3}}+d_{5} .
$$

From $d u / d p=-1$, by utilizing the value of $z$, we obtain

$$
\dot{x}=-\frac{\ln \left(t+d_{1}\right)}{d_{3}}+d_{5} .
$$

We deduce that

$$
x=\left(t+d_{1}\right) \ln \left(t+d_{1}\right)-d_{5} t+d_{6} .
$$

Thus (37), (40), and (42) are solutions of system (33).

(2) Consider the oscillatory motion of a system of three particles governed by

$$
\begin{aligned}
& \ddot{x}=k_{1} e^{-t k_{0}} \\
& \ddot{y}=-k_{2} \dot{y}^{2}+k_{0} \dot{y} \\
& \ddot{z}=-k_{2} \dot{z}^{2}+k_{0} \dot{z}
\end{aligned}
$$

in a region; $a_{1}<x<a_{2}, b_{1}<y<b_{2}$ and $c_{1}<z<c_{2}$ such that for any small number $\epsilon>0,\left|a_{1}-a_{2}\right|^{2}=\epsilon, \mid b_{1}-$ $\left.b_{2}\right|^{2}=\epsilon$ and $\left|c_{1}-c_{2}\right|^{2}=\epsilon$, where $\epsilon \rightarrow 0$ and $k_{0} ; k_{1}$ are arbitrary constants whereas $k_{2}$ is a drag constant. There is a time varying applied force which produces the motion after neglecting the effects of gravity due to small region. 
System (43) admits the Lie algebra $A_{3,4}^{3}$ spanned by symmetry generators

$$
\begin{aligned}
& X_{1}=k_{1} \frac{\partial}{\partial x} \\
& X_{2}=\frac{t k_{1}}{k_{0}} \frac{\partial}{\partial x} \\
& X_{3}=-\frac{1}{k_{0}} \frac{\partial}{\partial t}+k_{1} x \frac{\partial}{\partial x} .
\end{aligned}
$$

By using the invertible transformations

$$
\begin{aligned}
& \bar{t}=t k_{0}, \\
& \bar{x}=\frac{1}{k_{1}} x, \\
& \bar{y}=e^{k_{2} y}, \\
& \bar{z}=e^{k_{2} z},
\end{aligned}
$$

this Lie algebra is transformed into the canonical form of the Lie algebra $A_{3,4}^{3}$ mentioned in [8]. System (43) is transformed into

$$
\begin{aligned}
& \ddot{x}=e^{-t} \\
& \ddot{y}=\dot{y}, \\
& \ddot{z}=\dot{z} .
\end{aligned}
$$

System (46) is matched with corresponding canonical form by taking $f=h=1$ and $g=0$ in it, plugging with $A_{3,4}^{3}$ mentioned in Table 1 . The associated differential invariants and canonical representation are

$$
\begin{aligned}
p & =z, \\
q & =y, \\
u & =\dot{z}, \\
\frac{d q}{d p} & =\frac{\dot{y}}{\dot{z}}, \\
w & =e^{t} \ddot{x}, \\
\frac{d u}{d p} & =\frac{\ddot{z}}{\dot{z}}, \\
\frac{d^{2} q}{d p^{2}} & =\frac{\ddot{y}}{\dot{z}^{2}}-\frac{\dot{y} \ddot{z}}{\dot{z}^{3}} .
\end{aligned}
$$

Using invariant representations, inverting back into original variables and dropping the bars, we get

$$
\begin{aligned}
& x=k_{1} e^{-t / k_{0}}+k_{1} e_{1} \frac{t}{k_{0}}+k_{1} e_{2} \\
& y=\frac{1}{k_{2}} \log \left(e^{t / k_{0}}+e_{3} \frac{t}{k_{0}}+e_{4}\right), \\
& z=\frac{1}{k_{2}} \log \left(e^{t / k_{0}}+e_{5} \frac{t}{k_{0}}+e_{6}\right) .
\end{aligned}
$$

These are solutions of system (43).

\section{Conclusion}

In symmetry analysis of differential equations, integrability is an important part of the analysis. In the literature different approaches have been constructed by employing admitted symmetries of differential equations. The classical approach of reductions is not valid in usual way for systems of ordinary differential equations as used for scalar ordinary differential equations (see, e.g., $[5,6,12])$. In this research work, our focus is on systems of three $k$ th-order $(k \geq 2)$ ordinary differential equations that admit three-dimensional Lie algebras.

By utilizing the approach developed by Ayub et al. in $[8,9,13]$, the bases of differential invariants for system of three 2nd-order ODEs admitting three-dimensional Lie algebras which are constructed. This is Lie algebraic approach with special type of invariant representation, obtained by exploiting the interrelationships of the invariants. Moreover canonical forms associated with these invariants for system of three 2nd-order ODEs are obtained and listed in Table 1. Furthermore singularity in invariant structures has also been investigated. In addition an algorithm for construction of systems of three $k$ th-order $(k \geq 2)$ ordinary differential equations possessing three-dimensional Lie algebras is provided. Integrability of canonical forms presented in Table 1 is investigated in detailed manner. Illustrative examples from mechanics of system of particles are presented to explain the integrability of canonical forms. This classification will provide a framework for classification of system of three 2ndorder ODEs admitting higher dimensional Lie algebras.

Remarks 12. (i) For Table 1 notations are mentioned in Section 2.1 whereas $f, g, h, k$, and $\phi$ are arbitrary functions.

(ii) Both of algebras $A_{3,1}^{4}$ and $A_{3,8}^{1}$ possess the same set of invariants plugging with a single equation (are not associated with systems of three 2nd-order ODEs); the canonical forms are not obtained for them. For example $A_{3,1}^{4}$ corresponds to the following invariants $u=x, v=y, d v / d u=$ $\dot{y} / \dot{x}, d^{2} v / d u^{2}=-\ddot{x} \dot{y} / \dot{x}^{3}+\ddot{y} / \dot{x}^{2}$ and the related single equation, namely, $-\ddot{x} \dot{y} / \dot{x}^{3}+\ddot{y} / \dot{x}^{2}=g(u, v, d v / d u)$. The invariant representations of these cases are not listed in Table 1.

(iii) The algebras $A_{3,1}^{3}$ and $A_{3,5}^{3}$ possess singular invariants structure.

\section{Disclosure}

M. N. Qureshi is presently at Allama Iqbal Open University (AIOU), Islamabad, Pakistan.

\section{Conflicts of Interest}

The authors declare that they have no conflicts of interest.

\section{References}

[1] O. Gaponova and M. Nesterenko, "Systems of second-order ODEs invariant with respect to low-dimensional Lie algebras," Physics AUC, vol. 16, no. 2, pp. 238-256, 2006. 
[2] V. M. Gorringe and P. G. L. Leach, "Kepler's third law and the oscillator isochronism," American Journal of Physics, vol. 61, pp. 991-995, 1993.

[3] K. S. Govinder and P. G. Leach, "Integrability of generalized Ermakov systems," Journal of Physics. A. Mathematical and General, vol. 27, no. 12, pp. 4153-4156, 1994.

[4] N. H. Ibragimov, F. M. Mahomed, D. P. Mason, and D. Sherwell, Differential Equations and Chaos, New Age International (P) Limited, New Delhi, India, 1996.

[5] J. Olver Peter, Applications of Lie Groups to Differential Equations, Graduate Text in Mathematics, vol. 107, Springer, New York, NY, USA, 2nd edition, 1993.

[6] C. Wafo Soh and F. M. Mahomed, "Reduction of order for systems of ordinary differential equations," Journal of Nonlinear Mathematical Physics, vol. 11, no. 1, pp. 13-20, 2004.

[7] S. Lie, Theories der Transformation gruppen. 3 vols. (Originally published in Leipzig, 1888, 1890, 1893), vol. 1888, 1890, 1893, Chelsea publishing Company, New York, NY, USA, 2nd edition, 1970.

[8] M. Ayub, M. Khan, and F. M. Mahomed, "Second-order systems of ODEs admitting three-dimensional lie algebras and integrability," Journal of Applied Mathematics, vol. 2013, Article ID 147921, 2013.

[9] M. Ayub, F. M. Mahomed, M. Khan, and M. N. Qureshi, "Symmetries of second-order systems of ODEs and integrability," Nonlinear Dynamics. An International Journal of Nonlinear Dynamics and Chaos in Engineering Systems, vol. 74, no. 4, pp. 969-989, 2013.

[10] G. M. Mubarakzjanov, “On Solvable Lie algebras, Izv," Izv. Vys. Ucheb. Zaved. Matematika, vol. 32, no. 1, pp. 114-123, 1963 (Russian).

[11] R. O. Popovych, V. M. Boyko, M. O. Nesterenko, and M. W. Lutfullin, "Realizations of real low-dimensional Lie algebras," Journal of Physics. A. Mathematical and General, vol. 36, no. 26, pp. 7337-7360, 2003.

[12] C. Wafo Soh and F. M. Mahomed, "Canonical forms for systems of two second-order ordinary differential equations," Journal of Physics. A. Mathematical and General, vol. 34, no. 13, pp. 28832911, 2001.

[13] M. Ayub, S. Sadique, and F. M. Mahomed, "Singular invariant structures for Lie algebras admitted by a system of second-order ODEs," Applied Mathematics and Computation, vol. 281, pp. 137147, 2016.

[14] T. G. Mkhize, S. Moyo, and S. V. Meleshko, "Complete group classification of systems of two linear second-order ordinary differential equations: the algebraic approach," Mathematical Methods in the Applied Sciences, vol. 38, no. 9, pp. 1824-1837, 2015.

[15] S. Suksern, S. Moyo, and S. V. Meleshko, "Application of group analysis to classification of systems of three second-order ordinary differential equations," Mathematical Methods in the Applied Sciences, vol. 38, no. 18, pp. 5097-5113, 2015.

[16] S. Suksern and N. Sakdadech, "Criteria for system of three second-order ordinary differential equations to be reduced to a linear system via restricted class of point transformation," Applied Mathematics, vol. 5, no. 3, pp. 553-571, 2014. 


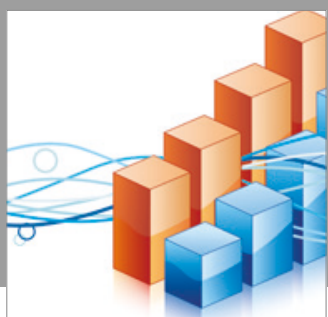

Advances in

Operations Research

vatersals

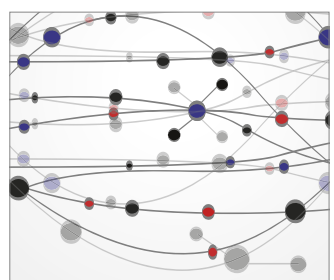

\section{The Scientific} World Journal
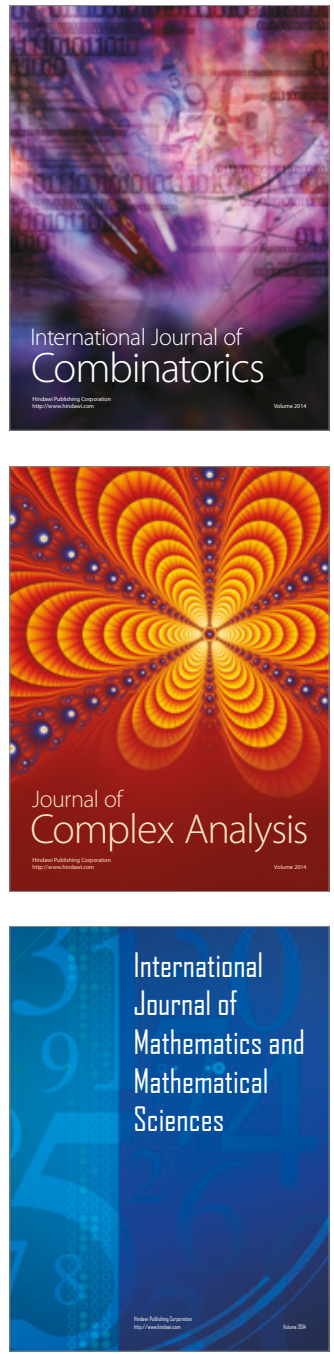
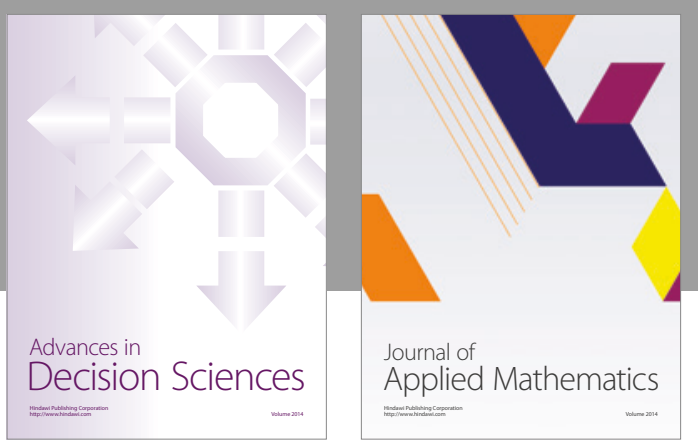

Algebra

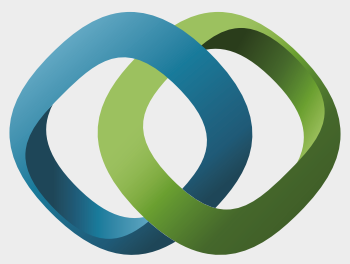

\section{Hindawi}

Submit your manuscripts at

https://www.hindawi.com
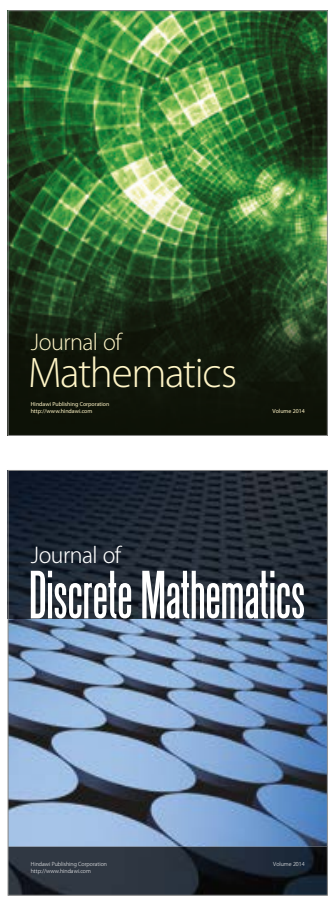

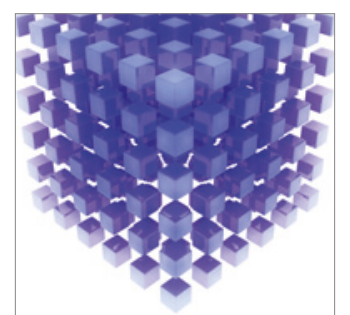

Mathematical Problems in Engineering
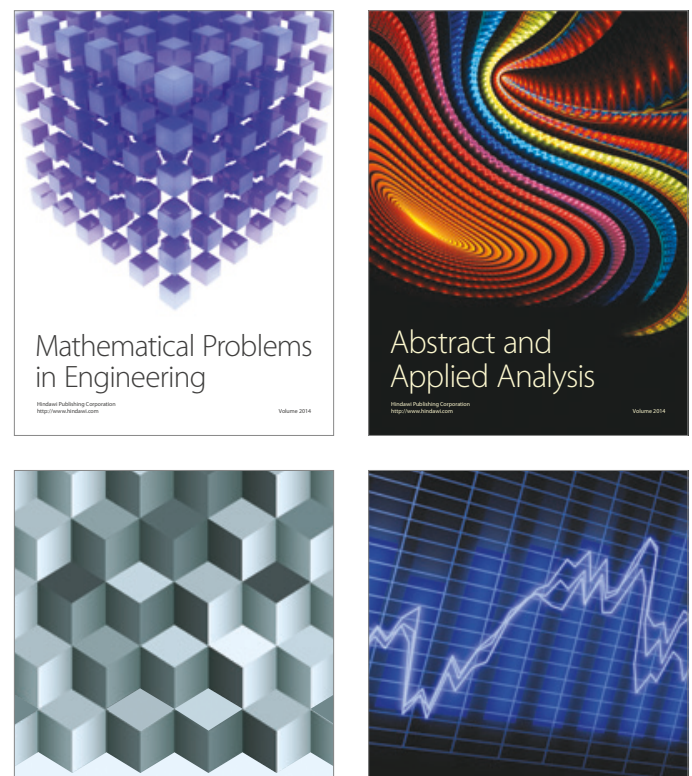

Journal of

Function Spaces

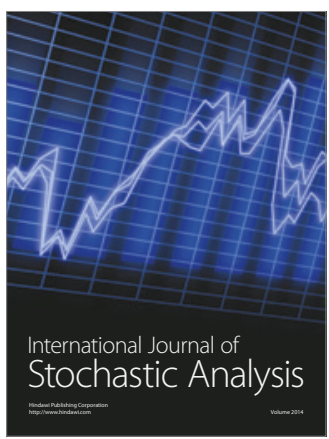

Probability and Statistics
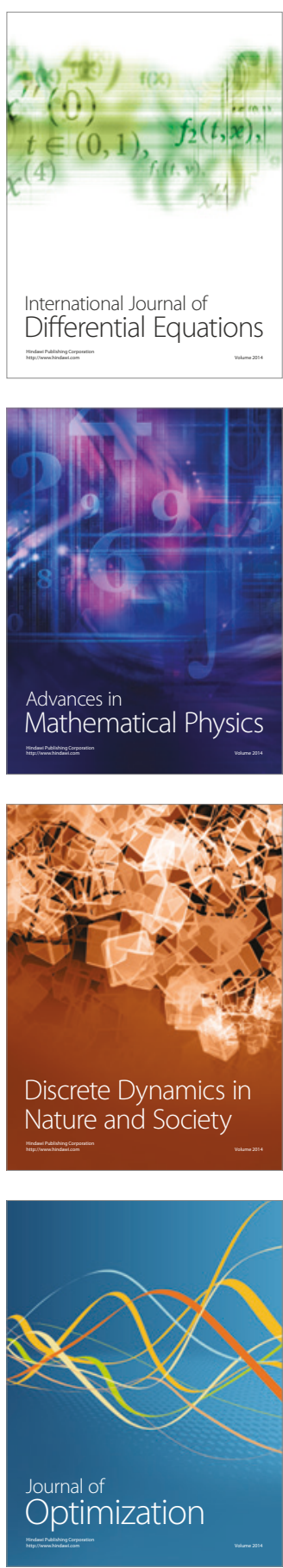